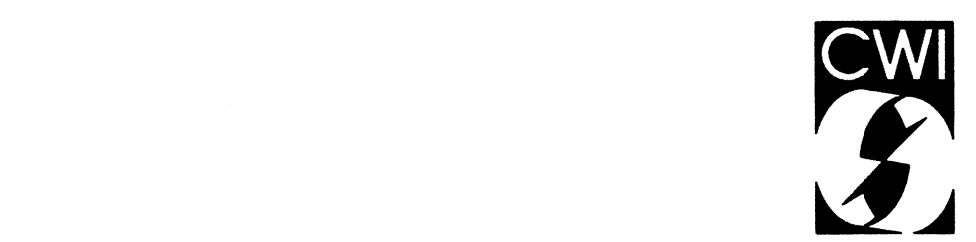

Centrum voor Wiskunde en Informatica Centre for Mathematics and Computer Science

J.W. Cohen

On the random walk with zero drifts in the first quadrant of $\mathbb{R}_{2}$ 
The Centre for Mathematics and Computer Science is a research institute of the Stichting Mathematisch Centrum, which was founded on February 11, 1946, as a nonprofit institution aiming at the promotion of mathematics, computer science, and their applications. It is sponsored by the Dutch Government through the Netherlands Organization for the Advancement of Research (N.W.O.).

Copyright $\odot$ Stichting Mathematisch Centrum, Amsterdam 


\title{
On the Random Walk with Zero Drifts in the First Quadrant of $\mathbb{R}_{2}$
}

\author{
J.W. Cohen \\ Centre for Mathematics and Computer Science \\ P.O. Box 4079, 1009 AB Amsterdam, The Netherlands \\ March 1990
}

\begin{abstract}
Summary
The random walk on the lattice in the positive quadrant is studied for the case that the one-step displacement vector has zero drift, finite second moments and support contained in $\{-1,0,1,2 \ldots\}$ $\times\{-1,0,1,2, \ldots\}$. It is well known that the first entrance time out from a point in the interior of the lattice into the union of the coordinate axies is finite with probability one. In the present study it is shown that its first moment is finite if and only if the covariance of the $x$ - and $y$-component of the one-step displacement vector is negative. For this case explicit expressions are given for the first moment of the entrance time and for the first and second moments of the hitting point of the axes, in terms of the second moment characteristics of the one-step displacement vector. The results are deduced from the hitting point identity for the random walk.
\end{abstract}

1980 Mathematics Subject Classification: 60J15

Keywords: Random walk, lattice, first quadrant, zero drift, entrance time into boundary, first moment, hitting points, hitting point identity.

\section{INTRODUCTION}

The random walk on the lattice with nonnegative, integer valued coordinates in $\mathbb{R}_{2}$ is studied for the case that the $x$ - and $y$-component of the one-step displacement vector have zero drift, and finite second moments, the support of the vector being contained in $\{-1,0,1, \ldots\} \times\{-1,0,1, \ldots\}$. It is well known that the first entrance time out from a point in the interior of the lattice into the set formed by the coordinate axes is finite with probability one. The present study concerns the first moment of this entrance time and the first and second moments of the hitting point at the coordinate axes.

The random walk $\mathbf{z}_{n} \equiv\left(\mathbf{x}_{n}, \mathbf{y}_{n}\right), n=0,1,2, \ldots$, with state space $S$, the lattice points with integer valued, nonnegative, coordinates in $\mathbb{R}_{2}$, i.e.

$$
S=\{0,1,2, \ldots\} \times\{0,1,2, \ldots\},
$$

is defined by: for $n=0,1,2, \ldots$,

i. for $\mathbf{x}_{n}>0, \mathbf{y}_{n}>0$ :

$$
\begin{aligned}
& \mathbf{x}_{n+1}=\mathbf{x}_{n}-1+\xi_{n}, \\
& \mathbf{y}_{n+1}=\mathbf{y}_{n}-1+\boldsymbol{\eta}_{n} ;
\end{aligned}
$$

ii. for $\mathbf{x}_{n}=0$ or $\mathbf{y}_{n}=0$,

$$
\begin{aligned}
& \mathbf{x}_{n+1}=\mathbf{x}_{n}, \\
& \mathbf{y}_{n+1}=\mathbf{y}_{n} ;
\end{aligned}
$$

iii. $\mathbf{x}_{0}=x_{0}, \mathbf{y}_{0}=y_{0}$;

with

i. the starting point

$$
z_{0} \equiv\left(x_{0}, y_{0}\right) \in S \text {, }
$$

ii. $\quad\left(\xi_{n}, \boldsymbol{\eta}_{n}\right), n=0,1,2, \ldots$, a sequence of i.i.d. vectors each with state space $S$ and

Report BS-R9022

Centre for Mathematics and Computer Science

P.O. Box 4079, 1009 AB Amsterdam, The Netherlands 


$$
\mathrm{E}\left\{\boldsymbol{\xi}_{n}\right\}=\mathrm{E}\left\{\boldsymbol{\eta}_{n}\right\}=1, \quad \mathrm{E}\left\{\boldsymbol{\xi}_{n}^{2}\right\}<\infty, \quad \mathrm{E}\left\{\boldsymbol{\eta}_{n}^{2}\right\}<\infty .
$$

Let $(\xi, \eta)$ be a stochastic vector with the same state space and bivariate distribution as $\left(\xi_{n}, \eta_{n}\right)$, i.e.

$$
(\xi, \eta) \sim\left(\xi_{n}, \eta_{n}\right)
$$

The bivariate generating function of the distribution of $(\xi, \eta)$ is defined by

$$
\phi\left(p_{1}, p_{2}\right):=\mathrm{E}\left\{p_{1}^{\xi} p_{2}^{\eta}\right\}, \quad\left|p_{1}\right| \leqslant 1,\left|p_{2}\right| \leqslant 1 .
$$

We introduce the

$$
\begin{aligned}
& \text { Assumption. } \\
& \text { i }\left|\phi\left(p_{1}, p_{2}\right)\right|=1 \text { for }\left|p_{1}\right|=1,\left|p_{2}\right|=1 \Rightarrow\left|p_{2}\right|=1,\left|p_{2}\right|=1 \text {; }
\end{aligned}
$$

ii. for every $(i, j) \in S$ the coefficient of $p_{1}^{i} p_{2}^{i}$ in the series expansion of $\left[\phi\left(p_{1}, p_{2}\right) / p_{1} p_{2}\right]^{n}$ with $\left|p_{1}\right|=1$; $\left|p_{2}\right|=1, n$ being a positive integer, is positive for $n$ sufficiently large;

iii. $\phi(0,1)>0, \phi(1,0)>0$.

REMARK 1.1. (1.5) $\mathrm{i}$ implies that the random walk $\mathbf{z}_{n}$ is aperiodic, cf. [1]; whereas (1.5) ii implies that its state space $S$ is irreducible; (1.5) iii is an obvious assumption, otherwise $\mathbf{x}_{n}$ and/or $\mathbf{y}_{n}$ cannot decrease.

Put

$$
B:=\{0\} \times\{0,1,2, \ldots\} \cup\{0,1,2, \ldots\} \times\{0\},
$$

so $B$ is the boundary of $S$.

Define

$$
\begin{aligned}
& \mathbf{m}\left(z_{0}\right):=\inf _{n=0,1 \ldots . .}\left\{n: \mathbf{z}_{n} \in B \mid z_{0} \in S\right\}, \\
& :=\infty \text { if } \mathbf{z}_{n} \notin B \text { for all } n=0,1,2, \ldots \text {; } \\
& \mathbf{k}\left(z_{0}\right) \equiv\left(\mathbf{k}_{1}\left(z_{0}\right), \mathbf{k}_{2}\left(z_{0}\right)\right):=\mathbf{z}_{\mathbf{m}\left(x_{0}\right)} \equiv\left(\mathbf{x}_{\mathbf{m}\left(z_{0}\right)}, \mathbf{y}_{\mathbf{m}\left(z_{0}\right)}\right) \text { if } \mathbf{m}\left(z_{0}\right)<\infty \text {, } \\
& :=(\infty, \infty) \quad, \quad=\infty .
\end{aligned}
$$

So $\mathbf{m}\left(z_{0}\right)$ is the first entrance time of the $\mathbf{z}_{n}$-processes into the boundary $B$ when starting at $z_{0}$, and $\mathbf{k}\left(z_{0}\right)$ is then the hitting point of $B$, note that

$$
\mathbf{k}_{1}\left(z_{0}\right) \mathbf{k}_{2}\left(z_{0}\right)=0 \text { if } \mathbf{m}\left(z_{0}\right)<\infty .
$$

The main result of the present study is the following

TheOREM 1.1. For $z_{0} \in S \backslash B$ :

i. $\operatorname{Pr}\left\{\mathbf{m}\left(z_{0}\right)<\infty\right\}=1$;

ii. $\quad \mathrm{E}\left\{\mathbf{k}_{1}\left(z_{0}\right)\right\}=x_{0}, \mathrm{E}\left\{\mathbf{k}_{2}\left(z_{0)}\right\}=y_{0}\right.$;

iii. $\mathrm{E}\{(\boldsymbol{\xi}-1)(\eta-1)\}<0 \Leftrightarrow \mathrm{E}\left\{\mathrm{m}\left(z_{0}\right)\right\}<\infty, \quad \mathrm{E}\left\{\mathbf{k}_{1}^{2}\left(z_{0}\right)\right\}<\infty, \quad \mathrm{E}\left\{\mathbf{k}_{2}^{2}\left(z_{0}\right)\right\}<\infty ;$

and for $\mathrm{E}\{(\xi-1)(\boldsymbol{\eta}-1)\}<0$,

$$
\begin{aligned}
& \text { i. } \quad \mathrm{E}\left\{\mathbf{m}\left(z_{0}\right)\right\}=\frac{x_{0} y_{0}}{-\mathrm{E}\{\xi-1)(\boldsymbol{\eta}-1)\}} ; \\
& \text { ii. } \left.\mathrm{E}\left\{\left(\mathbf{k}_{1}\left(z_{0}\right)-x_{0}\right)^{2}\right\}=\mathrm{E}\{\boldsymbol{\xi}-1)^{2}\right\} \mathrm{E}\left\{\mathbf{m}\left(z_{0}\right)\right\} \text {, } \\
& \mathrm{E}\left\{\left(\mathbf{k}_{2}\left(z_{0}\right)-y_{0}\right)^{2}\right\}=\mathrm{E}\left\{(\boldsymbol{\eta}-1)^{2}\right\} \mathrm{E}\left\{\mathbf{m}\left(z_{0}\right)\right\} \text {. }
\end{aligned}
$$


Theorem 1.1 is quite remarkable since it formulates explicitly in terms of the second moments of the one-step displacement vector $(\xi-1, \boldsymbol{\eta}-1)$ the necessary and sufficient condition for the first moment of the entrance time to be finite and further the expression for this moment as well as the expressions for the first and second moments of the hitting point. Actually (1.10) i is a wellknown result for twodimensional random walks for which (1.3) i applies, cf. [1].

The proof of theorem 1.1 is based on the hitting point identity for the $\mathbf{z}_{n}$-process, see below, and it is presented in section 2. The proof needs several asymptotic results, these are derived in the appendix, see section 3 .

ReMARK 1.2. Mostly we shall delete $z_{0}$ in the symbols defined in (1.7) and (1.8), i.e. we shall write for $z_{0} \in S \backslash B$,

$$
\mathbf{m} \equiv \mathbf{m}\left(z_{0}\right), \mathbf{k}_{1} \equiv \mathbf{k}_{1}\left(z_{0}\right), \quad \mathbf{k}_{2} \equiv \mathbf{k}_{2}\left(z_{0}\right) ;
$$

further $(A)$ shall denote the indicator function of the event $A$, i.e.

$$
\begin{aligned}
(A) & =1 \text { if } A \text { occurs, } \\
& =0, \bar{A}, \quad .
\end{aligned}
$$

The hitting point identity for the $\mathbf{z}_{n}$-process defined above reads: for $|r| \leqslant 1$,

$$
\hat{p}_{1}^{x_{0}} \hat{p}_{2}^{y_{0}}=\mathrm{E}\left\{r^{\mathbf{m}} \hat{p}_{1}^{\mathbf{k}_{1}}\left(\mathbf{k}_{1}>0\right)\right\}+\mathrm{E}\left\{r^{\mathbf{m}}\left(\mathbf{k}_{1}=\mathbf{k}_{2}=0\right)\right\}+\mathrm{E}\left\{r^{\mathbf{m}} \hat{p}_{2}^{\mathbf{k}_{2}}\left(\mathbf{k}_{2}>0\right)\right\},
$$

with for $|r| \leqslant 1, r \neq 1$,

$\left(\hat{p}_{1}, \hat{p}_{2}\right)$ any zero tuple of the kernel

$$
Z\left(r, p_{1}, p_{2}\right):=p_{1} p_{2}-r \phi\left(p_{1}, p_{2}\right), \quad\left|p_{1}\right| \leqslant 1,\left|p_{2}\right| \leqslant 1,
$$

and for $r=1$,

$\left(\hat{p}_{1}, \hat{p}_{2}\right)$ any zero tuple which is the limit for $r \rightarrow 1$ of a zero tuple $\left(\hat{p}_{1}, \hat{p}_{2}\right)$ of $Z\left(r, p_{1}, p_{2}\right)$ with $|r| \leqslant 1$, $r \neq 1$.

REMARK 1.3. $\left(\hat{p}_{1}, \hat{p}_{2}\right)$ is said to be a zero tuple of $Z\left(r, p_{1}, p_{2}\right)$ if

$$
Z\left(r, \hat{p}_{1}, \hat{p}_{2}\right)=0 \text {. }
$$

Remark 1.4. The H.P.I. (1.10) is a special case of a more general identity discussed in [2], viz. take in the notation of [2], $N=2, M=0$ and $T$ empty.

\section{Proof of THEOREM 1.1}

From lemma 3.1 it follows that $\left(\hat{p}_{1}, \hat{p}_{2}\right)$ with

$$
\hat{p}_{2}=p_{2},\left|p_{2}\right|=1, \quad \hat{p}_{1}=P_{1}\left(r, p_{2}\right),|r| \leqslant 1, \quad r \neq 1,
$$

is a zero tuple of $Z\left(r, p_{1}, p_{2}\right),\left|p_{1}\right| \leqslant 1,\left|p_{2}\right| \leqslant 1$, and that for $r \rightarrow 1$ it is also a zero tuple.

REMARK 2.1. By symmetry the zero tuple

$$
\left(p_{1}, P_{2}\left(r, p_{1}\right)\right), \quad\left|p_{2}\right|=1,
$$

is constructed.

Hence from (1.12) we have for $z_{0} \in S \backslash B,|r| \leqslant 1,\left|p_{2}\right|=1$,

$$
P_{1}^{x_{0}}\left(r, p_{2}\right) p_{2}^{y_{0}}=\mathrm{E}\left\{r^{\mathbf{m}} P_{1}^{\mathbf{k}_{1}}\left(r, p_{2}\right)\left(\mathbf{k}_{1} \geqslant 0, \mathbf{k}_{2}=0\right)\right\}+\mathrm{E}\left\{r^{\mathbf{m}} p_{2}^{\mathbf{k}_{2}}\left(\mathbf{k}_{2}>0\right)\right\} .
$$

Since $P_{1}(r, 1) \rightarrow 1$ for $r \rightarrow 1$ it follows from (2.2) by taking $p_{2}=1$ and letting $r \rightarrow 1$ that 


$$
1=\operatorname{Pr}\{\mathbf{m}<\infty\},
$$

i.e. (1.10) i has been proved. For $|r| \leqslant 1, r \neq 1,\left|p_{2}\right|=1$ we hase $\left|P_{1}\left(r, p_{2}\right)\right|<1$ so

$$
\left|\mathrm{E}\left\{r^{\mathbf{m}} \mathbf{k}_{1} P_{1}^{\mathbf{k}_{1}-1}\left(r, p_{2}\right)\right\}\right|<\infty ;
$$

since $P_{1}\left(r, p_{2}\right)$ possesses a derivative with respect to $p_{2}$, see lemma 3.1 ii, it follows that the last term in (2.2) possesses also a first derivative.

Differentiate (2.2) with respect to $p_{2}$ and then take $p_{2}=1$, it then follows for $|r| \leqslant 1, r \neq 1$,

$$
\left.\frac{\partial P_{1}\left(r, p_{2}\right)}{\partial p_{2}}\right|_{p_{2}=1}\left[x_{0} P_{1}^{x_{0}-1}(r, 1)-\mathrm{E}\left\{r^{\mathbf{m}} \mathbf{k}_{1} P_{1}^{\mathbf{k}_{1}-1}(r, 1)\right]+y_{0} P_{1}^{x_{0}}(r, 1)-\mathrm{E}\left\{r^{\mathbf{m}} \mathbf{k}_{2}\right\}=0\right.
$$

So by letting $r \rightarrow 1$ we obtain from (2.3) and (3.9),

$$
\begin{aligned}
& -\frac{\boldsymbol{\alpha}_{12}}{\boldsymbol{\alpha}_{1}}\left[x_{0}-\mathrm{E}\left\{\mathbf{k}_{1}\right\}\right]+y_{0}-\mathrm{E}\left\{\mathbf{k}_{2}\right\}=0, \\
& x_{0}-\mathrm{E}\left\{\mathbf{k}_{1}\right\}-\frac{\boldsymbol{\alpha}_{12}}{\boldsymbol{\alpha}_{2}}\left[y_{0}-\mathrm{E}\left\{\mathbf{k}_{2}\right\}\right]=0 ;
\end{aligned}
$$

where the second relation in (2.4) follows by symmetry, cf. remark 2.1 , from the first one. Because of (3.3) the main determinant of the set of equations is nonzero, so this system has only the zero solution, if $\mathrm{E}\left\{\mathbf{k}_{1}\right\}<\infty, \mathrm{E}\left\{\mathbf{k}_{2}\right\}<\infty$, and then (1.10) ii follows; for the ultimate proof see below, directly after (2.13), and note that that proof uses only (2.2) and (3.6).

By taking $p_{2}=1$ in (2.2) and differentiating with respect to $r$ we obtain for $|r| \leqslant 1, r \neq 1$,

$$
\begin{aligned}
& \frac{\mathrm{d} P_{1}(r, 1)}{\mathrm{d} r}\left[x_{0} P_{1}^{x_{0}-1}(r, 1)-\mathrm{E}\left\{r^{\mathbf{m}} \mathbf{k}_{1} P_{1}^{\mathbf{k}_{1}-1}(r, 1)\right\}\right]= \\
& \mathrm{E}\left\{\mathbf{m} r^{\mathbf{m}-1} P_{1}^{\mathbf{k}_{1}}(r, 1)\left(\mathbf{k}_{1} \geqslant 0, \mathbf{k}_{2}=0\right)\right\}+\mathrm{E}\left\{\mathbf{m} r^{\mathbf{m}-1}\left(\mathbf{k}_{2}>0\right)\right\} .
\end{aligned}
$$

From (2.3) and (2.5) it follows for $|r| \leqslant 1, r \neq 1$,

$$
\begin{aligned}
& \left.\frac{\partial P_{1}\left(r, p_{2}\right)}{\partial p_{2}}\right|_{p_{2}=1}\left[\mathrm{E}\left\{\mathbf{m} r^{\mathbf{m}-1}\left\{P_{1}^{\mathbf{k}_{1}}(r, 1)\left(\mathbf{k}_{1} \geqslant 0, \mathbf{k}_{2}=0\right)+\left(\mathbf{k}_{2}>0\right)\right\}\right\}\right]= \\
& -\frac{\mathrm{d} P_{1}(r, 1)}{\mathrm{d} r}\left[y_{0} P_{1}^{x_{0}}(r, 1)-\mathrm{E}\left\{r^{\mathbf{m}} \mathbf{k}_{2}\right\}\right] .
\end{aligned}
$$

We consider this relation for $|r| \leqslant 1, r \sim 1$, it then follows from (2.6), (3.5) ii, (3.9) and (1.10) ii that

$$
\begin{aligned}
& \left\{-\alpha_{12}+o(1)\right\} \mathrm{E}\left\{\mathbf{m} r^{\mathbf{m}-1}\left[P_{1}^{\mathbf{k}_{1}}(r, 1)\left(\mathbf{k}_{1} \geqslant 0, \mathbf{k}_{2}=0\right)+\left(\mathbf{k}_{2}>0\right)\right]\right\}= \\
& \left\{-2+\frac{1}{2} \sqrt{2} \frac{\alpha_{1}^{1 / 2}}{\sqrt{1-r}}\right\}\left(1-\frac{o(\sqrt{1-r})}{\sqrt{1-r}}\right)\left[y_{0}\left(1-P_{1}^{x_{0}}(r, 1)\right)-\mathrm{E}\left\{\mathbf{k}_{2}\left(1-r^{\mathbf{m}}\right)\right\}\right]+o(\sqrt{1-r})= \\
& -2 \sqrt{2} \sqrt{1-r} x_{0} y_{0} \alpha_{1}^{1 / 2}+2 \mathrm{E}\left\{\mathbf{k}_{2}\left(1-r^{\mathbf{m}}\right)\right\}+ \\
& {\left[x_{0} y_{0}-\frac{1}{2} \sqrt{2} \alpha_{1}^{1 / 2} \mathrm{E}\left\{\mathbf{k}_{2} \frac{1-r^{\mathbf{m}}}{\sqrt{1-r}}\right\}\right]\left(1-\frac{o(\sqrt{1-r})}{\sqrt{1-r}}\right) .}
\end{aligned}
$$

Suppose

$$
\alpha_{12}<0 \text {. }
$$

then for $r>0$ and $r \sim 1$ the lefthand side of (2.7) is positive, possibly $+\infty$ for $r \rightarrow 1$, and since

$$
\mathrm{E}\left\{\mathbf{k}_{2} \frac{1-r^{\mathbf{m}}}{\sqrt{1-r}}\right\}>0 \text { for } 0<r<1,
$$

it follows from (2.7) that 


$$
x_{0} y_{0} \sqrt{2} \alpha_{1}^{-1 / 2}>\limsup _{r \rightarrow 1} \mathrm{E}\left\{\mathbf{k}_{2} \frac{1-r^{\mathbf{m}}}{\sqrt{1-r}}\right\} \geqslant 0 .
$$

Hence the lefthand side of (2.7) has for $r \rightarrow 1$ a finite limit which since $P_{1}(r, 1) \rightarrow 1$ is equal to

$$
-\alpha_{12} E\{\mathbf{m}\}
$$

and hence $E\{\mathbf{m}\}$ should be finite, so we have shown that, cf. (2.8),

$$
\alpha_{12}<0 \Rightarrow \mathrm{E}\{\mathbf{m}\}<\infty,
$$

and moreover that the following limit exists and

$$
x_{0} y_{0} \sqrt{2} \alpha_{1}^{-1 / 2}>\lim _{r \rightarrow 1} \mathrm{E}\left\{\mathbf{k}_{2} \frac{1-r^{\mathbf{m}}}{\sqrt{1-r}}\right\} \geqslant 0 \text {. }
$$

Next we take in (2.2) $r=1$ and differentiate with respect to $p_{2}$, cf. lemma 3.1 iv, this yields for $\left|p_{2}\right|=1, p_{2} \neq 1$,

$$
\begin{aligned}
& \mathrm{E}\left\{\mathbf{k}_{1} P_{1}^{\mathbf{k}_{1}-1}\left(1, p_{2}\right)\right\} \frac{\mathrm{d} P_{1}\left(1, p_{2}\right)}{\mathrm{d} p_{2}}+\mathrm{E}\left\{\mathbf{k}_{2} p_{2}^{\mathbf{k}_{2}-1}\right\}= \\
& x_{0} P_{1}^{x_{0}-1}\left(1, p_{2}\right) p_{2}^{y_{0}} \frac{\mathrm{d} P_{1}\left(1, p_{2}\right)}{\mathrm{d} p_{2}}+y_{0} P_{1}^{x_{0}}\left(1, p_{2}\right) p_{2}^{y_{0}-1},
\end{aligned}
$$

and

$$
\begin{aligned}
& \mathrm{E}\left\{\mathbf{k}_{1}\left(\mathbf{k}_{1}-1\right) P_{1}^{\mathbf{k}_{1}-2}\left(1, p_{2}\right)\right\}\left\{\frac{\mathrm{d} P_{1}\left(1, p_{2}\right)}{\mathrm{d} p_{2}}\right\}^{2}+\mathrm{E}\left\{\mathbf{k}_{1} P_{1}^{\mathbf{k}_{1}-1}\left(1, p_{2}\right)\right\} \frac{\mathrm{d}^{2} P_{1}\left(1, p_{2}\right)}{\mathrm{d} p_{2}^{2}}+\mathrm{E}\left\{\mathbf{k}_{2}\left(\mathbf{k}_{2}-1\right) p_{2}^{\mathbf{k}_{2}-2}\right\}= \\
& x_{0}\left(x_{0}-1\right) P_{1}^{x_{0}-2}\left(1, p_{2}\right) p_{2}^{y_{0}}\left\{\left[\frac{\mathrm{d} P_{1}\left(1, p_{2}\right)}{\mathrm{d} p_{2}}\right]^{2}+x_{0} P_{1}^{x_{0}-1}\left(1, p_{2}\right) p_{2}^{y_{0}} \frac{\mathrm{d}^{2} P_{2}\left(1, p_{2}\right)}{\mathrm{d} p_{2}^{2}}+\right.
\end{aligned}
$$

$2 x_{0} y_{0} P_{1}^{x_{0}-1}\left(1, p_{2}\right) p_{2}^{y_{0}-1} \frac{\mathrm{d} P_{1}\left(1, p_{2}\right)}{\mathrm{d} p_{2}}+y_{0}\left(y_{0}-1\right) P_{1}^{x_{0}}\left(1, p_{2}\right) p_{2}^{y_{0}-2}$

as in the derivation of (2.5) it is readily shown that $\mathrm{E}\left\{p_{2}^{\mathbf{k}_{2}}\left(\mathbf{k}_{2}>0\right)\right\}$ is twice differentiable for $\left|p_{2}\right|=1$, $p_{2} \neq 1$. Letting $p_{2} \rightarrow 1$ in (2.11) yields since $P_{1}\left(1, p_{2}\right) \rightarrow 1$,

$$
\left.\left\{\mathrm{E}\left\{\mathbf{k}_{1}\right\}-x_{0}\right\} \frac{\mathrm{d} P_{1}\left(1, p_{2}\right)}{\mathrm{d} p_{2}}\right|_{p_{2}=1}=y_{0}-\mathrm{E}\left\{\mathbf{k}_{2}\right\} \text {. }
$$

From (3.3) and (3.6) it is seen that $\left.\frac{\mathrm{d} P_{1}\left(1, p_{2}\right)}{\mathrm{d} p_{2}}\right|_{p_{2}=1}$ is complex and hence we obtain (1.10) ii.

Suppose

$$
\mathrm{E}\left\{\mathbf{k}_{1}^{2}\right\}<\infty, \quad \mathrm{E}\left\{\mathbf{k}_{2}^{2}\right\}<\infty .
$$

It then follows from (2.12) that for $p_{2} \rightarrow 1$,

$$
\begin{aligned}
& {\left[\mathrm{E}\left\{\mathbf{k}_{1}\left(\mathbf{k}_{1}-1\right)\right\}-x_{0}\left(x_{0}-1\right)\right]\left\{\left.\frac{\mathrm{d} P_{1}\left(1, p_{2}\right)}{\mathrm{d} p_{2}}\right|_{p_{2}=1}\right\}^{2}+\mathrm{E}\left\{\mathbf{k}_{2}\left(\mathbf{k}_{2}-1\right)\right\}-y_{0}\left(y_{0}-1\right)} \\
& -\left.2 x_{0} y_{0} \frac{\mathrm{d} P_{1}\left(1, p_{2}\right)}{\mathrm{d} p_{2}}\right|_{p_{2}=1}=-\lim _{p_{2} \rightarrow 1}\left\{\left[\mathrm{E}\left\{\mathbf{k}_{1} P_{1}^{\mathbf{k}_{1}-1}\left(1, p_{2}\right)\right\}-x_{0} P_{1}^{x_{0}-1}\left(1, p_{2}\right) p_{2}^{y_{0}}\right] \frac{\mathrm{d}^{2} P_{1}\left(1, p_{2}\right)}{\mathrm{d} p_{2}^{2}}\right\},
\end{aligned}
$$

where the limit in the righthand side should exist since (2.14) and (3.6) i show that the left hand side is finite.

By using (1.10) ii, i.e. $\mathrm{E}\left\{\mathbf{k}_{1}\right\}=x_{0}$, it follows that for $p_{2} \rightarrow 1$,

$$
\mathrm{E}\left\{\mathbf{k}_{1} P_{1}^{\mathbf{k}_{1}-1}\left(1, p_{2}\right)\right\}-x_{0} P_{1}^{x_{0}-1}\left(1, p_{2}\right) p_{2}^{y_{0}} \rightarrow 0,
$$


and so since $\left.\frac{\mathrm{d} P_{1}\left(1, p_{2}\right)}{\mathrm{d} p_{2}}\right|_{p_{2}=1}$ is finite it follows from (2.14) that the left hand side of (2.16) behaves as $1-p_{2}$ for $p_{2} \sim 1,\left|p_{2}\right|=1$. Consequently, it follows from lemma 3.3. ii that the righthand side of (2.15) is equal to zero; by using (3.6) $\mathrm{i}$, or better (3.8), a simple calculation shows that (2.15) with its righthand side being zero may be rewritten as

$\left.2\left[\mathrm{E}\left\{\left(\mathbf{k}_{1}-x_{0}\right)^{2}\right\} \frac{\alpha_{12}}{\alpha_{1}}+x_{0} y_{0}\right] \frac{\mathrm{d} P_{1}\left(1, p_{2}\right)}{\mathrm{d} p_{2}}\right|_{p_{2}=1}=\mathrm{E}\left\{\left(\mathbf{k}_{2}-y_{0}\right)^{2}\right\}-\frac{\alpha_{2}}{\alpha_{1}} \mathrm{E}\left\{\left(\mathbf{k}_{1}-x_{0}\right)^{2}\right\}$.

Since $\left.\frac{\mathrm{d} P_{1}\left(1, p_{2}\right)}{\mathrm{d} p_{2}}\right|_{p_{2}=1}$ is complex it follows from (2.17) that

$$
\mathrm{E}\left\{\left(\mathbf{k}_{1}-x_{0}\right)^{2}\right\}=x_{0} y_{0} \frac{\alpha_{1}}{-\alpha_{12}}, \quad \mathrm{E}\left\{\left(\mathbf{k}_{2}-y_{0}\right)^{2}\right\}=x_{0} y_{0} \frac{\alpha_{2}}{-\alpha_{12}} .
$$

The lefthand sides in (2.18) are positive and consequently it has been shown that, cf. (2.14),

$$
\mathrm{E}\left\{\mathbf{k}_{1}^{2}\right\}<\infty, \mathrm{E}\left\{\mathbf{k}_{2}^{2}\right\}<\infty \Rightarrow \alpha_{12}<0 .
$$

To complete the proof of (1.10) iii it is seen from (2.9) and (2.19) that we have still to show that

$$
\mathrm{E}\{\mathbf{m}\}<\infty \Rightarrow \mathrm{E}\left\{\mathbf{k}_{1}^{2}\right\}<\infty, \mathrm{E}\left\{\mathbf{k}_{2}^{2}\right\}<\infty .
$$

To prove (2.20) we rewrite (2.5) for $|r| \leqslant 1, r \neq 1$ as

$$
\begin{aligned}
& \mathrm{E}\left\{\mathbf{m} r^{\mathbf{m}} P_{1}^{\mathbf{k}_{1}}(r, 1)\left(\mathbf{k}_{1} \geqslant 0, \mathbf{k}_{2}=0\right)\right\}+\mathrm{E}\left\{\mathbf{m} r^{\mathbf{m}-1}\left(\mathbf{k}_{2}>0\right)\right\}= \\
& \frac{\mathrm{d} P_{1}(r, 1)}{\mathrm{d} r}\left(1-P_{1}(r, 1)\right)\left[-x_{0} \frac{1-P_{1}^{x_{0}-1}(r, 1)}{1-P_{1}(r, 1)}+\mathrm{E}\left\{\mathbf{k}_{1} r^{\mathbf{m}} \frac{1-P_{1}^{\mathbf{k}_{1}-1}(r, 1)}{1-P_{1}(r, 1)}\right\}\right]+ \\
& \frac{\mathrm{d} P_{1}(r, 1)}{\mathrm{d} r} \sqrt{1-r} \mathrm{E}\left\{\mathbf{k}_{1} \frac{1-r^{\mathbf{m}}}{\sqrt{1-r}}\right\} .
\end{aligned}
$$

Hence by using the asymptotic relations (3.5) we have from (2.21) for $r \sim 1$,

$$
\begin{aligned}
& \mathrm{E}\left\{\mathbf{m} r^{\mathbf{m}} P_{1}^{\mathbf{k}_{1}}(r, 1)\left(\mathbf{k}_{1} \geqslant 0, \mathbf{k}_{2}=0\right)\right\}+\mathrm{E}\left\{\mathbf{m} r^{\mathbf{m}-1}\left(\mathbf{k}_{2}>0\right)\right\}= \\
& \mathrm{E}\left\{\mathbf{k}_{1} \frac{1-r^{\mathbf{m}}}{\sqrt{1-r}}\right\}\left\{-2 \alpha_{1}^{-1} \sqrt{1-r}+\frac{1}{2} \sqrt{2} \alpha^{-1 / 2}\left(1-\frac{o(\sqrt{1-r})}{\sqrt{1-r}}\right)+o(\sqrt{1-r})\right\}+ \\
& \left\{-2 \sqrt{2} \alpha_{1}^{-1 / 2} \sqrt{1-r}+\alpha_{1}^{-1}\left(1-\frac{o(\sqrt{1-r})}{\sqrt{1-r}}\right)\right\}-x_{0} \frac{1-P_{1}^{x_{0}-1}(r, 1)}{1-P_{1}(r, 1)}+\mathrm{E}\left\{\mathbf{k}_{1} r^{\mathbf{m}} \frac{1-P_{1}^{\mathbf{x}_{0}-1}(r, 1)}{1-P_{1}(r, 1)}\right\} .
\end{aligned}
$$

We next observe that (2.7) implies that

$\mathrm{E}\left\{\mathbf{m} r^{\mathbf{m}-1} P_{1}^{\mathbf{k}_{1}}(r, 1)\left(\mathbf{k}_{1} \geqslant 0, \mathbf{k}_{2}=0\right)\right\}+\mathrm{E}\left\{\mathbf{m} r^{\mathbf{m}-1}\left(\mathbf{k}_{2}>0\right)\right\}>0,0<r<1$.

and

$$
\mathrm{E}\left\{\mathbf{k}_{2} \frac{1-r^{\mathbf{m}}}{\sqrt{1-r}}\right\}>0, \quad 0<r<1,
$$

both have a limit for $r \rightarrow 1$ and these limits are both finite or both $+\infty$. So by symmetry, cf. remark 2.1 , the same holds for

$$
\mathrm{E}\left\{\mathbf{m} r^{\mathbf{m}-1} P_{2}^{\mathbf{k}_{2}}(r, 1)\left(\mathbf{k}_{2} \geqslant 0, \mathbf{k}_{2}=0\right)\right\}+\mathrm{E}\left\{\mathbf{m} r^{\mathbf{m}}\left(\mathbf{k}_{1}>0\right)\right\}
$$

and

$$
\mathrm{E}\left\{\mathbf{k}_{1} \frac{1-r^{\mathbf{m}}}{\sqrt{1-r}}\right\}
$$


Suppose

$$
\mathrm{E}\{\mathbf{m}\}<\infty,
$$

then by the observation just made it follows from (2.22) that the following limit should exits and be finite:

$$
\left|\lim _{r \rightarrow 1}\left\{\mathrm{E}\left\{\mathbf{k}_{1} r^{\mathbf{m}} \frac{1-P_{1}^{\mathbf{k}_{1}-1}(r, 1)}{1-P_{1}(r, 1)}\right\}-x_{0} \frac{1-P_{1}^{x_{0}-1}(r, 1)}{1-P_{1}(r, 1)}\right\}\right|<\infty .
$$

Since $\mathbf{k}_{1}\left(\mathbf{k}_{1}-1\right) \geqslant 0$ with probability one it follows that for $0<r<1$ and $r \rightarrow 1$,

$$
\begin{aligned}
& \mathrm{E}\left\{\mathbf{k}_{1} r^{\mathbf{m}} \frac{1-P_{1}^{\mathbf{k}-1}(r, 1)}{1-P_{1}(r, 1)}\right\} \leqslant \mathrm{E}\left\{\mathbf{k}_{1} \frac{1-P_{1}^{\mathbf{k}_{1}-1}(r, 1)}{1-P_{1}(r, 1)}\right\} \rightarrow \mathrm{E}\left\{\mathbf{k}_{1}\left(\mathbf{k}_{1}-1\right)\right\}, \\
& x_{0} \frac{1-P_{1}^{x_{0}-1}(r, 1)}{1-P_{1}(r, 1)} \rightarrow x_{0}\left(x_{0}-1\right),
\end{aligned}
$$

and consequently (2.23) implies that $\mathrm{E}\left\{\mathbf{k}_{1}^{2}\right\}$ should be finite, and by symmetry also $\mathrm{E}\left\{\mathbf{k}_{2}^{2}\right\}<\infty$. Hence (2.20) has been proved, and so is (1.10) iii.

To prove (1.11) let $r \rightarrow 1$ in (2.7) and (2.22) with $\alpha_{12}<0$ then it follows

$$
\begin{aligned}
-\alpha_{12} \mathrm{E}\{\mathbf{m}\} & =x_{0} y_{0}-\frac{1}{2} \sqrt{2} \alpha_{1}^{1 / 2} \lim _{r \rightarrow 1} \mathrm{E}\left\{\mathbf{k}_{2} \frac{1-r^{\mathbf{m}}}{\sqrt{1-r}}\right\}, \\
& =x_{0} y_{0}-\frac{1}{2} \sqrt{2} \alpha_{2}^{1 / 2} \lim _{r \rightarrow 1} \mathrm{E}\left\{\mathbf{k}_{1} \frac{1-r^{\mathbf{m}}}{\sqrt{1-r}}\right\}, \\
\mathrm{E}\{\mathbf{m}\} & =\frac{1}{2} \sqrt{2} \alpha_{1}^{-1 / 2} \lim _{r \rightarrow 1} \mathrm{E}\left\{\mathbf{k}_{1} \frac{1-r^{\mathbf{m}}}{\sqrt{1-r}}\right\}+\alpha_{1}^{-1} \mathrm{E}\left\{\left(\mathbf{k}_{1}-x_{0}\right)^{2}\right\},
\end{aligned}
$$

with the second relation in (2.26) based on symmetry. Elimination of the limit from (2.26) and (2.27) yields by using (2.18),

$$
\mathrm{E}\{\mathbf{m}\}=\frac{x_{0} y_{0}}{-\alpha_{12}}
$$

and

$$
\lim _{r \rightarrow 1} \mathrm{E}\left\{\mathbf{k}_{1} \frac{1-r^{\mathbf{m}}}{\sqrt{1-r}}\right\}=\lim _{r \rightarrow 1} \mathrm{E}\left\{\mathbf{k}_{2} \frac{1-r^{\mathbf{m}}}{\sqrt{1-r}}\right\}=0,
$$

and hence (1.11) follows from (2.27) and (2.28).

\section{APPENDIX}

For the proof of theorem 1.1 we need a number of properties of a special class of zero tuples of the kernel, i.e. for $|r| \leqslant 1$, of

$$
Z\left(r, p_{1}, p_{2}\right):=p_{1} p_{2}-r \mathrm{E}\left\{p_{1}^{\xi} p_{2}^{\eta}\right\}, \quad\left|p_{1}\right| \leqslant 1,\left|p_{2}\right| \leqslant 1,
$$

with, cf. (1.3) ii,

$$
\mathrm{E}\{\boldsymbol{\xi}\}=1, \quad \mathrm{E}\{\boldsymbol{\eta}\}=1, \quad \mathrm{E}\left\{\xi^{2}\right\}<\infty, \quad \mathrm{E}\left\{\boldsymbol{\eta}^{2}\right\}<\infty .
$$

Put

$$
\alpha_{1}:=\mathrm{E}\left\{(\xi-1)^{2}\right\}, \quad \alpha_{12}:=\mathrm{E}\{(\xi-1)(\eta-1)\}, \quad \alpha_{2}:=\mathrm{E}\left\{(\eta-1)^{2}\right\},
$$

so that, cf. (1.5),

$$
\alpha_{1} \alpha_{2}>\alpha_{12}^{2} \text {. }
$$


Lemma 3.1. $i$. The kernel $Z\left(r, p_{1}, p_{2}\right)$ has for $|r| \leqslant 1, r \neq 1,\left|p_{2}\right|=1$, a unique zero, say, $P_{1}\left(r, p_{2}\right)$ in $\left|p_{1}\right| \leqslant 1$;

ii. $P_{1}\left(1, p_{2}\right):=\lim _{r \rightarrow 1} P_{1}\left(r, p_{2}\right),\left|p_{2}\right|=1, p_{2} \neq 1$ is a unique zero of $Z\left(1, p_{1}, p_{2}\right)$ in $\left.\left|p_{1}\right|\right) \leqslant 1$,

$$
\begin{gathered}
\left|P_{1}\left(1, p_{2}\right)\right|<1 \text { for }\left|p_{2}\right|=1,\left|p_{2}\right| \neq 1, \\
P_{1}(1,1):=\lim _{p_{2} \rightarrow 1} P_{1}\left(1, p_{2}\right)=1,
\end{gathered}
$$

and all these zeros have multiplicity one except $P_{1}(1,1)$ which has multiplicity two.

iii. $P_{1}\left(r, p_{2}\right),|r| \leqslant 1, r \neq 1$ is a twice differentiable function of $p_{2}$ on $\left|p_{2}\right|=1$, the same applies for $P_{1}\left(1, p_{2}\right)$ on $\left|p_{2}\right|=1, p_{2} \neq 1$.

iv. $P_{1}\left(r, p_{2}\right),\left|p_{2}\right|=1$ is a regular function of $r$ for $|r| \leqslant 1, r \neq 1$.

PRoof. For fixed $p_{2}$ with $\left|p_{2}\right|=1$ it is easily seen that $\mathrm{E}\left\{p_{1}^{\xi} p_{2}^{\eta-1}\right\}$ is a regular function of $p_{1}$ for $\left|p_{1}\right|<1$, and continuous for $\left|p_{1}\right| \leqslant 1$. From (1.5) $\mathrm{i}$ it is seen for $|r| \leqslant 1, r \neq 1,\left|p_{2}\right|=1$ that on $\left|p_{1}\right|=1$,

$$
\left|p_{1}\right|=1>|r|\left|\mathrm{E}\left\{p_{1}^{\xi} p_{2}^{\eta-1}\right\}\right|
$$

so that by applying Rouche's theorem the first statement follows, and $P_{1}\left(r, p_{2}\right),|r| \leqslant 1, r \neq 1,\left|p_{2}\right|=1$ is a single zero and is nonzero for $r \neq 0$, cf. (1.5) iii. Obviously, $P_{1}\left(r, p_{2}\right),|r|<1,\left|p_{2}\right|=1$ is a continuous function of $r$ since $Z\left(r, p_{1}, p_{2}\right)$ is such a function, so $Z\left(1, p_{1}, p_{2}\right),\left|p_{2}\right|=1$ can have atmost one zero in $\left|p_{1}\right|<1$, and since for $\left|p_{1}\right|=1, p_{1} \neq 1,\left|p_{2}\right|=1$,

$$
\left|p_{1}\right|>\left|\mathrm{E}\left\{p_{1}^{\xi} p_{2}^{\eta-1}\right\}\right|
$$

it follows that $P_{1}\left(1, p_{2}\right)$ is the only zero of $Z\left(1, p_{1}, p_{2}\right),\left|p_{2}\right|=1, p_{2} \neq 1$ in $\left|p_{1}\right| \leqslant 1$, it has multiplicity one and is nonzero, cf. (15) iii. Obviously $p_{1}=1$ is a zero of $Z\left(1, p_{1}, 1\right)$, so that the continuity of $Z\left(1, p_{1}, p_{2}\right)$ in $p_{1}, p_{2}$ on $\left|p_{1}\right| \leqslant 1,\left|p_{2}\right|=1$ implies that $P_{1}\left(1, p_{2}\right) \rightarrow 1$ for $p_{2} \rightarrow 1$. Because $\mathrm{E}\{\xi\}=1$ and $P_{1}(r, 1)$ is positive for $0<r \leqslant 1$ it is readily verified that $P_{1}(1,1)$ is a zero with multiplicity two, note that

$$
\lim _{r \rightarrow 1} P_{1}(r, 1)=\lim _{p_{2} \rightarrow 1} P_{1}\left(1, p_{2}\right) .
$$

The third statements follows directly from the fact that $Z\left(r, p_{1}, p_{2}\right)$ possess a derivative with respect to $p_{1}$ for $\left|p_{1}\right| \leqslant 1$, and similarly with respect to $p_{2}$ for $\left|p_{2}\right| \leqslant 1$, and the fact that $P_{1}\left(r, p_{2}\right),\left|p_{2}\right|=1$ and $P_{1}\left(1, p_{2}\right),\left|p_{2}\right|=1, p_{2} \neq 1$ are zeros with multiplicity one. The proof of the ivth statement is similar.

REMARK 3.1. Denote by $\mathbf{m}_{1}$ for the component random walk $\mathbf{x}_{n}, n=0,1, \ldots$, cf. (1.2), with $x_{0}=1$ the first entrance time into the zero state. Simple arguments from the theory of one dimensional random walks lead directly to

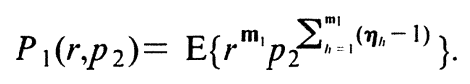

LEMMA 3.2. For $|r| \leqslant 1, r \sim 1$,

$$
\begin{aligned}
& \text { i. } P_{1}(r, 1)=1-\sqrt{2} \alpha_{1}^{-1 / 2} \sqrt{1-r}+o(\sqrt{1-r}), \\
& \text { ii. } \frac{\mathrm{d} P_{1}(r, 1)}{\mathrm{d} r}=-2 \alpha_{1}^{-1}+\frac{1}{2} \sqrt{2} \alpha_{1}^{-1 / 2} \frac{1}{\sqrt{1-r}}\left(1-\frac{o(\sqrt{1-r})}{\sqrt{1-r}}\right)+O(\sqrt{1-r}) .
\end{aligned}
$$

Proof. For $p_{2}=1$ it follows from (3.1) and lemma 3.1 for $|r| \leqslant 1, r \neq 1$, since $P_{1}(1,1)=1$ and 
$\alpha_{1}=\mathrm{E}\left\{(\xi-1)^{2}\right\}<\infty$ that with $r \sim 1$,

$$
\begin{aligned}
& 1-\left(1-P_{1}(r, 1)\right)=r \mathrm{E}\left\{\left[1-\left(1-P_{1}(r, 1)\right)\right]^{\xi}\right\}= \\
& r \mathrm{E}\{1\}-r \mathrm{E}\{\xi\}\left(1-P_{1}(r, 1)\right)+\frac{r}{2} \mathrm{E}\{\xi(\xi-1)\}\left(1-P_{1}(r, 1)\right)^{2}+o\left(\left(1-P_{1}(r, 1)\right)^{2}\right),
\end{aligned}
$$

or with $\mathrm{E}\{\boldsymbol{\xi}\}=1$,

$$
\frac{1}{2} r \alpha_{1}\left(1-P_{1}(r, 1)\right)^{2}+(1-r)\left(1-P_{1}(r, 1)\right)-(1-r)+o\left(\left(1-P_{1}(r, 1)^{2}\right)=0,\right.
$$

i.e.

$$
\frac{1}{2} r \alpha_{1}\left\{\frac{1-P_{1}(r, 1)}{\sqrt{1-r}}\right\}^{2}+\sqrt{1-r} \frac{1-P_{1}(r, 1)}{\sqrt{1-r}}-1+o\left(\left(\frac{1-P_{1}(r, 1)}{\sqrt{1-r}}\right)^{2}\right)=0 .
$$

Hence it follows that

$$
\frac{1-P_{1}(r, 1)}{\sqrt{1-r}}=O(1) \text { for } r \sim 1,
$$

and so, note that $1-P_{1}(r, 1)>0$ for $0<r<1$,

$$
\frac{1-P_{1}(r, 1)}{\sqrt{1-r}}=\frac{1}{r \alpha_{1}}\left\{-\sqrt{1-r}+\sqrt{1-r+2 r \alpha_{1}}\right\}+O(\sqrt{1-r}),
$$

and (3.5) i follows.

Obvious $P_{1}(r, 1)$ has a derivative for $|r| \leqslant 1, r \neq 1$, and by using the fact that $P_{1}(r, 1)$ is a zero of $Z\left(r, p_{1}, 1\right)$ it follows readily for $|r| \leqslant 1, r \neq 1$,

$$
\frac{\mathrm{d} P_{1}(r, 1)}{\mathrm{d} r} \mathrm{E}\left\{(1-\xi) P_{1}^{\xi}(r, 1)\right\}=\frac{1}{r} \mathrm{E}\left\{P_{1}^{\xi+1}(r, 1)\right\}
$$

or

$$
\frac{\mathrm{d} P_{1}(r, 1)}{\mathrm{d} r} \mathrm{E}\left\{(1-\xi)\left[1-\left(1-P_{1}(r, 1)\right)\right]^{\xi}\right\}=\frac{1}{r} \mathrm{E}\left\{\left[1-\left(1-P_{1}(r, 1)\right)\right]^{\xi+1}\right\} .
$$

Hence since $\mathrm{E}\left\{\xi^{2}\right\}<\infty$ and $\mathrm{E}\{\boldsymbol{\xi}\}=1$, we have for $|r| \leqslant 1, r \neq 1, r \sim 1$,

$$
\begin{aligned}
& \frac{\mathrm{d} P_{1}(r, 1)}{\mathrm{d} r}\left[\mathrm{E}\{(\xi-1) \xi\}\left(1-P_{1}(r, 1)\right)+o\left(1-P_{1}(r, 1)\right)\right]=\frac{1}{r}\left(1-\mathrm{E}\{(\xi+1)\}\left(1-P_{1}(r, 1)\right)\right)+ \\
& \frac{1}{2 r} \mathrm{E}\{(\xi+1) \xi\}\left(1-P_{1}(r, 1)\right)^{2}+o\left(\left(1-P_{2}(r, 1)\right)^{2}\right) .
\end{aligned}
$$

so

$$
\frac{\mathrm{d} P_{1}(r, 1)}{\mathrm{d} r}\left[\alpha_{1}+\frac{o\left(1-P_{1}(r, 1)\right)}{1-P_{1}(r, 1)}\right]=\frac{1}{r} \frac{1}{1-P_{1}(r, 1)}-\frac{2}{r}+\frac{\alpha_{1}+2}{2 r}\left(1-P_{1}(r, 1)\right)+o\left(1-P_{1}(r, 1)\right) .
$$

Inserting (3.5) $\mathrm{i}$ in the latter relation leads directly to

$$
\frac{\mathrm{d} P_{1}(r, 1)}{\mathrm{d} r}\left[1+\frac{o(\sqrt{1-r})}{\sqrt{1-r}}\right]=\frac{1}{2} \sqrt{2} \alpha_{1}^{-1 / 2} \frac{1}{\sqrt{1-r}}-\frac{2}{\alpha_{1}}+O(\sqrt{1-r}),
$$

or

$$
\frac{\mathrm{d} P_{1}(r, 1)}{\mathrm{d} r}=\frac{1}{2} \sqrt{2} \alpha_{1}^{-1 / 2} \frac{1}{\sqrt{1-r}}\left\{1-\frac{o(\sqrt{1-r})}{\sqrt{1-r}}\right\}-\frac{2}{\alpha_{1}}+O(\sqrt{1-r})
$$

LEMMA 3.3. 
i. $\left.\quad \frac{\mathrm{d} P_{1}\left(1, p_{2}\right)}{\mathrm{d} p_{2}}\right|_{p_{2}=1}:=\lim _{p_{2} \rightarrow 1} \frac{\mathrm{d} P_{1}\left(1, p_{2}\right)}{\mathrm{d} p_{2}}=\frac{1}{\alpha_{1}}\left\{-\alpha_{12} \pm \sqrt{\alpha_{12}^{2}-\alpha_{1} \alpha_{2}}\right\}$,

where the \pm sign corresponds to $p_{2}=e^{ \pm i \phi}$ for $\phi \rightarrow 0$;

ii. for $\left|p_{2}\right|=1, p_{2} \neq 1$ and $p_{2} \rightarrow 1$,

$$
\left(1-p_{2}\right) \frac{\mathrm{d}^{2} P_{1}\left(1, p_{2}\right)}{\mathrm{d} p_{2}^{2}} \rightarrow 0 \text {. }
$$

Proof. With $p_{2}=e^{i \phi},-\pi \leqslant \phi \leqslant \pi$, it follows readily from lemma 3.1. iii and

$$
\left[1-\left(1-p_{2}\right)\right]\left[1-\left(1-P_{1}\left(1, p_{2}\right)\right)\right]=\mathrm{E}\left\{\left[1-\left(1-P_{1}\left(1, p_{2}\right)\right)\right]^{\xi}\left[1-\left(1-p_{2}\right)\right]^{\eta}\right\}=0,
$$

that for $\phi \rightarrow \pm 0$,

$\alpha_{1}\left(1-P_{1}\left(1, p_{2}\right)\right)^{2}{ }_{-} \alpha_{12}\left(1-p_{2}\right)\left(1-P_{1}\left(1, p_{2}\right)\right)+\alpha_{2}\left(1-p_{2}\right)^{2}+o\left(\left(1-P_{1}\left(1, p_{2}\right)\right)^{2}\right)+o\left(\left(1-p_{2}\right)^{2}\right)=0$.

Hence

$$
\frac{1-P_{1}\left(1, p_{2}\right)}{1-p_{2}}
$$

has a limit for $\phi \downarrow 0$ as well as for $\phi \uparrow 0$. For $\left|p_{2}\right|=1, p_{2} \neq 1$ we have from lemma 3.1,

$$
\frac{\mathrm{d} P_{1}\left(1, p_{2}\right)}{\mathrm{d} p_{2}}=-\frac{P_{1}\left(1, p_{2}\right)}{p_{2}} \frac{\left.\mathrm{E}\{1-\eta) P_{1}^{\xi}\left(1, p_{2}\right) p_{2}^{\eta}\right\}}{\mathrm{E}\left\{(1-\xi) P_{1}^{\xi}\left(1, p_{2}\right) p_{2}^{\eta}\right\}} \text {. }
$$

Obviously $p_{2}=1$ is a zero of the numerator as well as at the denominator in (3.7), hence for $p_{2} \rightarrow 1$,

$$
\left.\frac{\mathrm{d} P_{1}\left(1, p_{2}\right)}{\mathrm{d} p_{2}}\right|_{p_{2}=1}=-\frac{\left.\mathrm{E}\{(1-\eta) \xi\} \frac{\mathrm{d} P_{1}\left(1, p_{2}\right)}{\mathrm{d} p_{2}}\right|_{p_{2}=1}+\mathrm{E}\{(1-\boldsymbol{\eta}) \boldsymbol{\eta}\}}{\left.\mathrm{E}\{(1-\xi) \xi\} \frac{\mathrm{d} P_{1}\left(1, p_{2}\right)}{\mathrm{d} p_{2}}\right|_{p_{2}=1}+\mathrm{E}\{(1-\xi) \eta\}} ;
$$

from which it follows

$$
\alpha_{1}\left[\left.\frac{\mathrm{d} P_{1}\left(1, p_{2}\right)}{\mathrm{d} p_{2}}\right|_{p_{2}=1}\right]^{2}+\left.2 \alpha_{12} \frac{\mathrm{d} P_{1}\left(1, p_{2}\right)}{\mathrm{d} p_{2}}\right|_{p_{2}=1}+\alpha_{2}=0,
$$

and the latter relation leads directly to (3.6) $\mathrm{i}$.

From (3.6) $\mathrm{i}$ it follows that $p_{2}=1$ is a zero with multiplicity one of the numerator and denominator in (3.7). Since, cf. (3.6),

$$
\begin{aligned}
& \left.\frac{\mathrm{d}}{\mathrm{d} p_{2}} \mathrm{E}\left\{(1-\xi) P_{1}^{\xi}\left(1, p_{2}\right) p_{2}{ }^{\eta}\right\}\right|_{p_{2}=1}=\left.\mathrm{E}\{(1-\xi) \xi\} \frac{\mathrm{d} P_{1}\left(1, p_{2}\right)}{\mathrm{d} p_{2}}\right|_{p_{2}=1}+\mathrm{E}\{(1-\xi) \eta\} \\
& =\mp \sqrt{\alpha_{12}^{2}-\alpha_{1} \alpha_{2}} \neq 0,
\end{aligned}
$$

it is seen that (3.6) ii holds.

LEMMA 3.4. For $|r| \leqslant 1, r \sim 1$,

$$
\left.\frac{\partial P_{1}\left(r, p_{2}\right)}{\partial p_{2}}\right|_{p_{2}=1}=-\frac{\alpha_{12}}{\alpha_{1}}+o(1) \text {. }
$$

Proof. From : for $|r| \leqslant 1,\left|p_{2}\right|=1$, 


$$
p_{2} P_{1}\left(r, p_{2}\right)=r \mathrm{E}\left\{P_{1}^{\xi}\left(r, p_{2}\right) p_{2}^{\eta}\right\},
$$

it follows readily, cf. lemma 3.1 iv that for $|r| \leqslant 1, r \neq 1,\left|p_{2}\right|=1$,

$$
p_{2} \frac{\partial P_{1}\left(r, p_{2}\right)}{\partial p_{2}}=-P_{1}\left(r, p_{2}\right) \frac{\mathrm{E}\left\{(1-\eta) P_{1}^{\xi}\left(r, p_{2}\right) p_{2}{ }^{\eta}\right\}}{\mathrm{E}\left\{(1-\xi) P_{1}^{\xi}\left(r, p_{2}\right) p_{2}{ }^{\eta}\right\}},
$$

so for $p_{2}=1$,

$$
\left.\frac{\partial P_{1}\left(r, p_{2}\right)}{\partial p_{2}}\right|_{p_{2}=1}=-P_{1}(r, 1) \frac{\mathrm{E}\left\{(1-\eta) P_{1}^{\xi}(r, 1)\right\}}{\mathrm{E}\left\{(1-\xi) P_{1}^{\xi}(r, 1)\right\}} .
$$

Hence for $|r| \leqslant 1, r \sim 1$, by using (3.5) i,

$$
\begin{aligned}
\frac{\partial P_{1}\left(r, p_{2}\right)}{\partial p_{2}} & =-\left[1-\left(1-P_{1}(r, 1)\right)\right] \frac{\mathrm{E}\left\{(1-\eta)\left[1-\left(1-P_{1}(r, 1)\right)\right]^{\xi}\right\}}{\left.\mathrm{E}\left\{(1-\xi)\left[1-P_{1}(r, 1)\right)\right]^{\xi}\right\}} \\
& =-\left[1-\left(1-P_{1}(r, 1)\right)\right] \frac{\mathrm{E}\{(1-\eta) \xi\}\left(1-P_{1}(r, 1)\right)+o\left(1-P_{1}(r, 1)\right)}{\mathrm{E}\left\{(1-\xi) \xi\left(1-P_{1}(r, 1)\right)+o\left(1-P_{1}(r, 1)\right)\right.} \\
& =-\frac{\alpha_{12}}{\alpha_{1}}+o(1) .
\end{aligned}
$$

\section{REFERENCES}

[1] SpitZer, F., Principles of Random Walks, D. van Nostrand Company, Princeton, 1964.

[2] CoHeN, J.W., On entrance times of a homogeneous $N$-dimensional random walk: an identity, in: A Celebration of Applied Probability, ed. J. Gani, Appl. Prob. Trust, Sheffield, England, 1988.

Acknowledgement. The author is indebted to his colleague Prof. A.J. Stam for some helpful remarks. 